\title{
AS CONCEPÇÕES DO CUIDADO GERONTOLÓGICO DE ENFERMAGEM FRENTE ÀS COMPLICAÇÕES PÓS-OPERATÓRIAS DO IDOSO
}

Maria Helena Lenardt ${ }^{1}$, Dâmarys Kohlbeck de Melo Neu², Susanne Elero Betiolli ${ }^{3}$, Márcia Daniele Seima ${ }^{4}$, Tatiane Michel ${ }^{5}$

RESUMO: O objetivo do estudo foi identificar concepções de cuidado ao idoso, concebidas pela equipe de enfermagem, frente às complicações no período pós-operatório. Trata-se de pesquisa qualitativa descritiva, realizada em uma clínica cirúrgica de um hospital de ensino de Curitiba, Paraná. Foram sujeitos do estudo dez profissionais de enfermagem segundo critérios estabelecidos para a participação. As informações foram coletadas por meio de entrevista semiestruturada e analisadas pelo método do Discurso do Sujeito Coletivo. Emergiram deste estudo seis ideias centrais: 1) Complicação cirúrgica é intercorrência inesperada no pós-operatório, desencadeada pelo procedimento cirúrgico. 2a) As complicações respiratórias sugerem agravos. 2b) Complicações relacionadas à ferida operatória são frequentes. 3a) Cuidado não diferenciado, realizado com ajuda do acompanhante. 3b) Modo de cuidar paternalista e feição infantilizada. 4) Cuidado é prioritário durante a higiene e no padrão de atenção curativista. Consideram-se incipientes as concepções de cuidado gerontológico de enfermagem, frente às complicações pós-operatórias do idoso.

PALAVRAS-CHAVE: Idoso; Complicações pós-operatórias; Enfermagem geriátrica; Cuidados de enfermagem.

\section{GERIATRIC NURSING CARE CONCEPTIONS REGARDING TO POST-OPERATIVE COMPLICATIONS OF THE ELDERLY}

\begin{abstract}
The study objective was to identify conceptions of the elderly care, designed by the nursing staff, regarding to complications in the postoperative period. It is a descriptive and qualitative study, conducted in a surgical clinic of a teaching hospital in Curitiba, Paraná. The subjects of the study were ten nursing professionals according to established criteria for participation. Information was collected through semi-structured interviews and analyzed by the collective subject discourse technique. Six core ideas emerged from this study: 1) surgical complication is unexpected complications in the postoperative period, triggered by the surgical procedure. 2a) Respiratory complications suggest illnesses. 2b) Complications related to surgical wounds are common. 3a) Non-specific Care, performed with help from the companion. 3b) A paternal caring and childish face. 4) Caring is a priority during hygiene and on the curative care standard. Conceptions of geriatric nursing care are considered as incipient, regarding the postoperative complications in the elderly.
\end{abstract}

KEYWORDS: Elderly; Postoperative complications; Geriatric nursing; Nursing care.

\section{LAS CONCEPCIONES DEL CUIDADO GERONTOLÓGICO DE ENFERMERÍA DELANTE DE LAS COMPLICACIONES POSOPERATORIAS DEL ANCIANO}

\begin{abstract}
RESUMEN: El objetivo del estudio fue identificar concepciones de cuidado al anciano, concebidas por el equipo de enfermería, delante de las complicaciones en periodo posoperatorio. Es una investigación cualitativa descriptiva, realizada en una clínica quirúrgica de un hospital de enseñanza de Curitiba, Paraná. Fueron sujetos del estudio diez profesionales de enfermería según criterios establecidos para la participación. Las informaciones fueron recogidas por medio de entrevista semiestructurada y analizadas por el método del Discurso del Sujeto Colectivo. Emergieron de este estudio seis ideas centrales: 1a) Complicación quirúrgica es ocurrencia inesperada en posoperatorio, desencadenada por el procedimiento quirúrgico. 2a) Las complicaciones respiratorias sugeren agravios. 2b) Complicaciones relacionadas a la herida operatoria son frecuentes. 3a) Cuidado no diferenciado, realizado con ayuda del acompañante. 3a) Modo de cuidar paternalista y aspecto infantilizado. 4) Cuidado es prioritario durante la higiene y en patrón de atención curativista. Se consideran incipientes las concepciones de cuidado gerontológico de enfermería, delante de las complicaciones posoperatorias del anciano.
\end{abstract}

PALABRAS CLAVE: Anciano; Complicaciones posoperatorias; Enfermería geriátrica; Cuidados de enfermería.

\footnotetext{
${ }^{1}$ Enfermeira. Doutora em Enfermagem. Professora Sênior do Programa de Pós-Graduação em Enfermagem da Universidade Federal do Paraná-UFPR. Líder do Grupo Multiprofissional de Pesquisa sobre Idosos-GMPI.

${ }^{2}$ Acadêmica do curso de Graduação em Enfermagem pela UFPR. Bolsista PIBIC/CNPq. Membro do GMPI.

${ }^{3}$ Acadêmica do curso de Graduação em Enfermagem pela UFPR. Bolsista PIBITI/TN. Membro do GMPI.

${ }^{4}$ Enfermeira. Mestranda do Programa de Pós-Graduação em Enfermagem da UFPR. Bolsista Reuni/UFPR. Membro do GMPI.

${ }^{5}$ Enfermeira. Mestranda do Programa de Pós-Graduação em Enfermagem da UFPR. Bolsista CAPES/UFPR. Membro do GMPI.
}

Autor correspondente:

Dâmarys Kohlbeck de Melo Neu

Universidade Federal do Paraná

Rua André Ferreira Camargo, 364 - 81710-100 - Curitiba-PR, Brasil

Recebido: $14 / 04 / 10$

Email: damy_neu@yahoo.com.br

Aprovado: 04/05/10

Cogitare Enferm. 2010 Jul/Set; 15(3):420-6 


\section{INTRODUÇÃO}

A tecnologia avançada oferece suporte às equipes para a realização de procedimentos cirúrgicos dentre a população idosa. $\mathrm{O}$ aumento da longevidade humana, acrescido deste avanço tecnológico e do desenvolvimento técnico-científico, contribuem para que um maior número de idosos busque procedimentos cirúrgicos como forma de tratamento ${ }^{(1)}$. É fato, porém, que as alterações fisiológicas decorrentes do envelhecimento podem influenciar negativamente a recuperação do paciente idoso. Os mais altos riscos cirúrgicos, nos pacientes com mais de 60 anos, têm associação com a evidência de complicações operatórias ${ }^{(2)}$.

A literatura traz como definição do termo complicação, aquilo que denota estado ou caráter do que é complicado, que apresenta dificuldade ${ }^{(3)}$. Neste estudo, entende-se por complicação cirúrgica no idoso aqueles eventos indesejáveis ocorridos no período pós-operatório imediato e mediato, que efetivamente dificultam a resolução dos impactos gerados pelo procedimento cirúrgico.

Estudo realizado com 2448 idosos submetidos à cirurgia após fratura de fêmur identificou a infecção pulmonar e a insuficiência cardíaca como complicações pós-operatórias mais comuns, responsáveis por elevar as taxas de mortalidade ${ }^{(4)}$. Em estudo retrospectivo, foram avaliados 124 prontuários de pacientes idosos submetidos a procedimento cirúrgico e observou-se que desses, $67,7 \%$ dos idosos apresentaram uma ou mais complicações pós-operatórias ${ }^{(5)}$.

As complicações no período pós-operatório podem ocorrer por diversos motivos. Por consequência, para os hospitais há elevação dos custos no que compete ao uso de antibióticos de última geração e ao aumento do tempo de hospitalização. Ainda, as complicações tornam-se injúrias ao paciente idoso, além de aumentarem as taxas de morbidade e mortalidade nesta faixa etária.

Os idosos apresentam elevado número de doenças crônicas passíveis de tratamento cirúrgico, no entanto, frequentemente, possuem reserva orgânica diminuída para reagir a um procedimento agressivo. $\mathrm{O}$ controle dos distúrbios orgânicos favorece a tolerância do idoso ao trauma cirúrgico ${ }^{(1)}$. Conhecer as condições do idoso e estar preparado para cuidar diante das possíveis complicações no período pós-operatório é um modo de evitar a gravidade da situação.

Com vistas à redução de risco cirúrgico, é imprescindível que a equipe de enfermagem acompanhe o paciente idoso, desde sua avaliação pré-operatória até a realização dos cuidados pós-operatórios. Para desenvolver um cuidado gerontológico de enfermagem, qualificado e resolutivo, há que se pensar na maneira pela qual a equipe de enfermagem identifica no idoso as possibilidades de complicações pós-operatórias. Nesse sentido, o objetivo deste estudo é identificar as concepções de cuidado ao idoso da equipe de enfermagem, frente às complicações no período pós-operatório.

\section{METODOLOGIA}

Trata-se de pesquisa qualitativa descritiva, realizada em clínica cirúrgica de um hospital de ensino de grande porte da cidade de Curitiba, Paraná. Participaram do estudo dez membros da equipe de enfermagem, sendo todos do sexo feminino. Desses, três são enfermeiras, uma é técnica de enfermagem, e seis são auxiliares de enfermagem.

Foram estabelecidos os seguintes critérios de inclusão para o estudo: ser efetivo ou contratado pela instituição para exercer as atividades de enfermeiro, técnico ou auxiliar de enfermagem; atuar profissionalmente na clínica cirúrgica há pelo menos três meses; aceitar a participação, preencher e assinar o termo de consentimento livre e esclarecido; e, autorizar a gravação das entrevistas.

As informações foram coletadas durante o mês de maio de 2008, por meio de entrevista semiestruturada composta de perguntas abertas. Foram abordadas as seguintes questões: O que é complicação cirúrgica? Quais as complicações cirúrgicas mais frequentes no idoso? Como realiza o cuidado aos pacientes idosos que apresentam complicações cirúrgicas? Quando presta cuidado ao idoso referente à complicação cirúrgica? As entrevistas foram gravadas em fitas magnéticas, e transcritas posteriormente.

Para análise das informações, empregou-se a técnica do Discurso do Sujeito Coletivo (DSC) $)^{(6)}$. Nesta abordagem, o DSC corresponde à descrição de um discurso que representa a compilação das partes essenciais dos discursos de cada participante do estudo, que embora apresentado na primeira pessoa do singular, configura-se como um discurso coletivo. As participantes da pesquisa que contribuíram para a formação do DSC estão identificadas na sequência por meio de códigos que variam de S1 a S10, para cada profissional. Os resultados foram apresentados em redação descritiva.

Foram respeitados os preceitos éticos de par- 
ticipação voluntária e consentida, segundo a resolução 196/96. Após acordo e consentimento dos representantes da clínica cirúrgica, o projeto foi encaminhado ao Comitê de Ética do Hospital para análise e parecer, o qual obteve aprovação sob o n. 1390.054/2007-03.

\section{RESULTADOS E DISCUSSÕES}

A idade das entrevistadas oscilou entre $25 \mathrm{e}$ 54 anos e o tempo de trabalho na área de enfermagem de quatro meses a 20 anos.

Das respostas obtidas nas entrevistas emergiram seis ideias centrais: 1) Complicação cirúrgica é intercorrência inesperada no período pós-operatório, desencadeada pelo procedimento cirúrgico. 2a) As complicações respiratórias sugerem agravos. 2b) As complicações relacionadas à ferida operatória são frequentes. 3a) Cuidado não diferenciado, realizado com ajuda do acompanhante. 3b) Modo de cuidar paternalista e feição infantilizada. 4) Cuidado é prioritário durante a higiene e no padrão de atenção curativista.

\section{O que é complicação cirúrgica?}

Ideia Central 1: Complicação cirúrgica é intercorrência inesperada no período pós-operatório desencadeada pelo procedimento cirúrgico.

DSC: Complicação cirúrgica é tudo que acontece no pós-operatório, que não é esperado; é tudo que sai do planejado para aquela cirurgia. São as intercorrências que acontecem quando a cirurgia não está dando certo. Alguma coisa que houve na cirurgia. Um paciente pós-cirúrgico que não se recupera dentro do prazo esperado. O primeiro dia no pós-operatório ele passa bem e depois você percebe que vai complicando. (S1, S2, S3, S7, S8 e S9)

As complicações ocorrem por diversos motivos e, geralmente, estão associadas ao preparo pré-operatório inadequadro $^{(7)}$. Conforme o DSC, complicação pós-operatória foi definida como algo inesperado, que acontece após um procedimento cirúrgico, portanto, sugere que essas situações aparecem de maneira súbita. No entanto, sabe-se que para a realização dos cuidados é necessário planejamento e organização. Por tal motivo, mesmo as situações não habituais ou imprevistas devem ser consideradas ao se elaborar o planejamento do cuidado, a fim de antever justamente o imprevisto.
A possibilidade de complicações pósoperatórias deve estar sempre à mente de todos os profissionais de saúde envolvidos no procedimento cirúrgico $^{(8)}$. É preciso estar atento aos sinais que indicam complicações, com a finalidade de atuar preventivamente, desde o período pré-operatório. Para apreender essa atenção, é imprescindível a aplicação de instrumentos de avaliação específicos para o idoso, preconizados pela gerontologia. O objetivo é proporcionar diagnóstico precoce do deficit, e identificar os fatores de risco, a fim de trazer maiores possibilidades para o cuidado efetivo diante do inesperado.

A utilização de protocolos de avaliação do idoso pré-cirúrgico é fundamental na tentativa de redução das complicações, bem como no direcionamento das ações de enfermagem para cada paciente. O planejamento deve estar centrado no idoso que será submetido à cirurgia, por meio de planos de cuidados específicos, o que auxilia a implementação de medidas preventivas que reduzem risco de iatrogenias e morte.

\section{Quais as complicações cirúrgicas mais frequentes no idoso?}

\section{Ideia Central 2a: As complicações respiratórias sugerem agravos.}

DSC: No idoso nós temos as respiratórias, como a pneumonia; às vezes, o paciente não consegue voltar da anestesia e acaba ficando no respirador. Isso evolui para a UTI, o paciente vem contaminado e complica cada vez mais. (S1, S3, S8 e S9)

As complicações respiratórias foram identificadas como as mais frequentes entre os idosos submetidos a procedimentos cirúrgicos. Estudos ${ }^{(9-12)}$ corroboram com o evidenciado pela equipe entrevistada. Essas complicações nos pacientes se devem a uma série de fatores associados, principalmente à presença de patologias pulmonares e à diminuição das reservas fisiológicas decorrentes do envelhecimento.

As alterações dos mecanismos reguladores da respiração são frequentes na pessoa idosa e ocasionam diminuição da resposta ventilatória às variações das pressões parciais de oxigênio arterial e do gás carbônico ${ }^{(13)}$. A anestesia, mencionada no DSC, é um dos fatores que predispõem às alterações respiratórias no pós-operatório, capaz de ocasionar hipoxemia, principalmente em idosos ${ }^{(12)}$. Essa condição pode se apresentar como confusão e agitação, e quando não controlada, faculta complicações graves como 
lesões neurológicas e cardiovasculares. Embora as complicações respiratórias sejam importantes fatores de aumento da morbidade e mortalidade no pós operatório de idosos, desenvolvida por $26,6 \%$ dos idosos em estudo retrospectivo de 124 prontuários ${ }^{(4)}$, não se pode deixar de tomar consciência das múltiplas facetas que caracterizam o processo de envelhecimento e que clamam para a necessidade de propiciar à pessoa idosa atenção preventiva abrangente.

\section{Ideia Central 2b: Complicações relacionadas à ferida operatória são frequentes.}

DSC: No idoso, o que a gente mais percebe são as feridas infectadas. Às vezes, uma hérnia incisional pós-cirúrgico, [...] complicação em idoso é bem importante, principalmente deiscência de sutura, ferimento; a cicatrização é mais prolongada; o idoso é bem mais complicado. (S1, S2, S3, S6 e S10)

Conforme o DSC, as complicações relacionadas à ferida operatória são as mais mencionadas. Essas complicações são comuns no pós-operatório dos idosos, porém não se destacam como as mais evidenciadas nesses pacientes, ocupando o terceiro lugar dentre as topografias mais frequentes de infecção hospitalar no idoso, com prevalência de $23,6 \%$ das infecções ${ }^{(9)}$.

Fatores como a realização de procedimentos invasivos, somados ao declínio da resposta imunológica e às alterações fisiológicas do envelhecimento, propiciam maior suscetibilidade do idoso em desenvolver uma infecção no período pós-operatório ${ }^{(14)}$.

As participantes do estudo apontaram deiscência de sutura como uma complicação comum da ferida operatória. Esta complicação é definida como a separação das camadas fasciais no início do pósoperatório, uma grande preocupação por se tratar de um evento que leva a uma reoperação ${ }^{(8)}$.

A observância aos sinais e sintomas que qualificam o início de uma deiscência de sutura, principalmente em sítio cirúrgico, é fundamental para que a intervenção dos cuidados seja precoce no sentido de evitar que o idoso seja levado a outro procedimento cirúrgico. Esse cuidado torna-se relevante, principalmente ao considerar as alterações do sistema imune do idoso que ainda está na condição pós-operatória, com o objetivo de evitar maiores sobrecargas e consequentes riscos para outras complicações.

Como realiza o cuidado aos pacientes idosos que apresentam complicações?
Ideia Central 3a: Cuidado não diferenciado, realizado com ajuda do acompanhante.

DSC: Os mesmos cuidados que a gente faz para o idoso a gente faz para o jovem. Então, não tem diferença no cuidado de enfermagem para o idoso. São os cuidados básicos, normal de pós-operatório. Não tem uma rotina que diga que o cuidado com o idoso é diferente do cuidado com o jovem. Na realidade, o idoso requer um cuidado diferenciado; isso seria uma coisa muito importante aqui na unidade. Seria muito adequado ter, porque a unidade tem muito mais paciente idoso e não acontece essa diferença que deveria ter. Então eu deixo acompanhante, porque é um direito dele. Com o acompanhante ao lado, a enfermagem fica mais tranquila e, qualquer intercorrência, o acompanhante vem correndo falar. Isso já é uma ajuda para nós. (S1, S2, S4, S5, S8, S9 e S10)

Verifica-se, no DSC, que não há distinção entre o cuidado de enfermagem prestado ao jovem, adulto ou idoso. A equipe de enfermagem entende que o idoso, em razão das alterações características do envelhecimento, requer cuidados específicos, porém não reconhece quais são estes cuidados.

Inicialmente, o cuidado gerontológico exige, por parte da enfermagem, a avaliação integral do idoso, especialmente quando este é submetido a procedimentos cirúrgicos. É fundamental reconhecer, com precisão, as alterações que levaram o idoso à cirurgia, particularmente se este estiver em situação de risco, a fim de instituir cuidados dirigidos aos idosos mais susceptíveis de complicação operatória. Essa avaliação prioritária requer o desenvolvimento de conhecimentos, tolerância e paciência da equipe de enfermagem, com o intuito de identificar os diversos problemas apresentados pelos idosos.

É preciso reconhecer que o idoso requer cuidados individualizados, personalizados e planejados pela enfermeira, em função de suas características particulares. Deste modo, "o cuidado gerontológico objetiva a promoção da saúde por meio de medidas que ajudem os idosos a se manterem saudáveis"(15:14).

Em estudo recente ${ }^{(16)}$, os integrantes da equipe de enfermagem referiram não ter conhecimento suficiente para a sua plena atuação profissional com os idosos. O conhecimento gerontológico é determinante para uma prática de enfermagem segura; sem ele, torna-se custoso garantir o cuidado especializado ao paciente idoso.

Pressupõe-se que a não-diferenciação dos 
cuidados e o deficit de conhecimentos no âmbito da gerontologia impulsionaram a equipe de enfermagem a recorrer ao auxílio dos acompanhantes. A permissão do acompanhante é um direito que consta no Estatuto do Idoso ${ }^{(17)}$, embora esse acompanhante também seja considerado como uma ajuda para o profissional. $\mathrm{O}$ deficit de recursos humanos constatado no hospital justifica esta atitude de aproximação, de ajuda no cuidado por parte do acompanhante.

Com vistas a essa situação, é primordial utilizar a observação atenta da equipe de enfermagem no que se refere às possíveis transferências de responsabilidade da mesma para os acompanhantes. Para a recuperação do idoso hospitalizado, é importante a presença do acompanhante, porém, é preciso a consciência de que, nessa situação, a responsabilidade pelos cuidados ao idoso é da equipe de enfermagem.

\section{Ideia Central 3b: Modo de cuidar paternalista e feição infantilizada.}

DSC: No pós-operatório imediato é a gente mesmo que faz, o paciente não consegue se ajudar muito. A partir do segundo dia a gente estimula, dai o que ele conseguir, ele mesmo vai fazer. Têm pacientes com dificuldade para deambular, aí você tem que estimular, tirar do leito, incentivar a alimentação, dar carinho, atenção. Geralmente, eles têm muita falta de carinho. [...] Aí, se você tratar eles como se fossem uma criança, fica mais fácil. A pessoa idosa, às vezes, se torna quase que sem defesa, igual uma criança. Então, a gente dá atenção para eles de maneira mais humanizada, e com um carinho especial. É um jeitinho todo diferente. Tendo o respeito pela pessoa idosa, conversando, explicando a situação um pouquinho mais, porque às vezes ela tem dificuldade de entendimento ou de audição. Não é nem explicar, às vezes é mesmo convencer. Basicamente, é orientação. (S2, S3, S5, S6 e S7)

Constata-se no DSC que o estímulo e a atenção prestada aos idosos são importantes na realização do cuidado, em razão do deficit auditivo e de entendimento que esses pacientes podem apresentar. Todavia, essas atitudes ocorrem de maneira equivocada, refletindo-se na infantilização do idoso. Proporcionar a feição de cuidado infantil ao idoso retira sua personalidade como adulto e é fator de desvalorização, além de acarretar consequências como aumento da dependência, perda da confiança em si e da dignidade como pessoa ${ }^{(18)}$.

Humanizar a relação terapêutica não é si- nônimo de tratamento infantilizado e, tampouco, paternalista. Ela é caracterizada pelo interesse (num amplo sentido), que o profissional desenvolve pelo idoso, agregado a uma série de conhecimentos gerontológicos que humanizam, de fato, o relacionamento profissional-paciente. As relações de empatia são fundamentais, porém isoladas não bastam para o cuidado gerontológico efetivo.

Conhecer e compreender o paciente idoso internado em clínica cirúrgica pode significar diferença decisiva na obtenção de cooperação e aceitação do mesmo quanto às recomendações de cuidado. $\mathrm{A}$ co-responsabilidade do cuidado é fundamental em gerontologia e, para tanto, os profissionais devem estimular nos idosos os sentimentos de confiança mútua e poder compartilhado, dentro de uma aliança terapêutica ${ }^{(19)}$.

É imprescindível estabelecer um vínculo com o paciente, e a explicação detalhada dos procedimentos funciona como engrenagem para a realização do cuidado, pois incentiva e reforça sua importância. Entretanto, deve-se ter clareza de que "o cuidado do idoso é muito mais do que "dar atenção maior"'(20:41).

$\mathrm{O}$ cuidado à saúde do idoso exige pautar-se na integralidade, com vistas à promoção, prevenção, proteção e reabilitação das condições de saúde, conforme preconiza o Ministério da Saúde ${ }^{(21)}$. No sentido da integralidade, os profissionais de saúde precisam abandonar o tecnicismo e o "modelo paternalista" de cuidado, que não se configuram efetivamente como terapêutico e ampliam seu papel para a implementação da interdisciplinaridade ${ }^{(22)}$.

\section{Quando presta cuidado ao idoso referente à com- plicação cirúrgica?}

\section{Ideia Central 4a: Cuidado é prioritário durante a higiene e no padrão de atenção curativista.}

DSC: A gente ajuda sempre no primeiro banho no leito, nunca deixa mesmo o acompanhante sozinho. O idoso não consegue fazer a higiene, a barba; muitas vezes chamamos voluntários para cortar o cabelo dos pacientes mais idosos que estão há muito tempo acamados. Assim que a complicação se apresenta, a gente normalmente atende o paciente e, se o médico não viu ainda a situação, mostramos o que aconteceu. Você chega e vê que a ferida tem alguma coisa, está hiperemiada, com bastante secreção. E quando acontece temos que fazer os cuidados. Ai é o tratamento. (S1, S2, S3, S4, S5, S6 e S10) 
A concepção de que o cuidado referente às complicações é percebido e realizado durante a higiene, justifica-se por ser o momento em que o profissional está mais próximo ao idoso. A equipe de enfermagem entende que é necessário sempre auxiliar no primeiro banho do pós-operatório. Desta forma, alguns pacientes recebem o auxílio para as ações de autocuidado sem que sejam diagnosticadas as necessidades para tais ações; são realizadas pela equipe de enfermagem por ser rotina na unidade, e não pela investigação da complicação cirúrgica. $\mathrm{O}$ cuidado gerontológico requer avaliação das características de cada paciente, com a finalidade de intervir quando preciso, além de estimular a participação do idoso no autocuidado, considerando que frequentemente este possui condições de executá-lo.

A realização da higiene permite à enfermagem ficar mais próxima ao paciente, e possibilita a oportunidade de avaliação por meio da observação e exame físico. Neste momento, a enfermeira pode estabelecer vínculos importantes para a realização dos demais cuidados, decorrentes da hospitalização do idoso em situação cirúrgica. O cuidado não pode ser compreendido apenas como procedimento de higiene em si, mas como uma oportunidade do olhar atento para a avaliação da evolução pós-operatória. Esse olhar perfaz todos os momentos, em todas as horas da hospitalização.

Os modos de cuidar em gerontologia perpassam a qualificação do cuidado, por meio da transformação do modelo tecnoassistencial para o de inovação, que proporciona o cuidado integral. Desse modo, a higiene corporal do idoso deve ser entendida como a oportunidade para diagnosticar as capacidades e as possibilidades nas atividades cotidianas do idoso e estimular as práticas da independência e autonomia, durante toda internação. Estimular essas práticas visa a inserção do idoso como sujeito ativo na sua comunidade, dentro das possibilidades de cada um.

Conforme afirma o DSC, o cuidado é realizado pela equipe de enfermagem, à medida que o idoso apresenta sinais de complicações. A prevenção efetiva demanda ações de cuidados anteriores à instalação da sintomatologia. $\mathrm{O}$ processo de trabalho alicerçado no modelo curativista não abre espaço para o desenvolvimento de competências para a prevenção e promoção da saúde. O ser idoso, pelas características biofisiológicas e sociais (estas principalmente em nosso país), exige o cuidado fundamentado nos princípios da integralidade, e não apenas o olhar sobre a doença e ou cirurgia.
Enfocar a assistência apenas nas complicações operatórias, depois de instaladas, descaracteriza o cuidado gerontológico de enfermagem. A enfermagem gerontológica atende o idoso em todos os níveis, desde a prevenção e promoção da saúde até a reabilitação ${ }^{(23)}$ e destaca-se num processo específico baseado na compreensão de parâmetros físicos, emocionais e de ordem social do idoso ${ }^{(24)}$.

\section{CONSIDERAÇÕES FINAIS}

Frente às complicações no período pós-operatório, consideram-se incipientes as concepções da equipe de enfermagem sobre o cuidado gerontológico no âmbito da assistência prestada ao idoso hospitalizado. Alguns cuidados, considerados específicos, são realizados durante a higiene e depois de instalada a complicação operatória, o que descaracteriza o cuidado gerontológico alicerçado no planejamento de ações preventivas.

Os resultados desta pesquisa e as crescentes estatísticas de idosos submetidos a procedimentos cirúrgicos são alertas para a necessidade de aperfeiçoamento dos profissionais que cuidam da população idosa. É notória a necessidade de capacitação dos profissionais, por meio de estudos gerontológicos, que visem melhorar a assistência prestada. Esses estudos resultam em planos de cuidados protocolados e específicos à pessoa idosa e são necessários para a consolidação da implementação de medidas preventivas que reduzam o risco de complicações e de iatrogenias. Planejar o cuidado gerontológico do pós-operatório possibilita reconhecer e refletir a intrínseca relação entre saber e fazer em Enfermagem.

\section{REFERÊNCIAS}

1. Santos Jr JCM. O paciente cirúrgico idoso. Rev Bras Coloproctol. 2003;23(4):305-16.

2. Petroianu A. Cirurgia em idosos. Rev Col Bras Cir. 2008;35(3):153-4.

3. Ferreira ABH. Novo dicionário da língua portuguesa. Rio de Janeiro: Nova Fronteira; 2004.

4. Roche JJW, Wenn RT, Sahota O, Moran CG. Effect of comorbidities and postoperative complications on mortality after hip fracture in elderly people: prospective observational cohort study. BMJ, 2005;55(1):331:6.

5. Borghi ACS. As complicações pós-operatórias 
do paciente idoso e as implicações ao cuidado de enfermagem gerontológico [dissertação]. Curitiba (PR): Universidade Federal do Paraná; 2007.

6. Lefèvre F, Lefèvre AMC, Teixeira JJV. O discurso do sujeito coletivo: uma nova abordagem metodológica em pesquisa qualitativa. Caxias do Sul: EDUCS; 2000.

7. Christóforo BEB, Carvalho DS. Cuidados de enfermagem realizados ao paciente cirúrgico no período préoperatório. Rev Esc Enferm USP. 2009;43(1):11-22.

8. Dayton MT. Complicações cirúrgicas. In: Townsend CM. Sabiston - tratado de cirurgia: a base biológica da moderna prática cirúrgica. v1. $17^{\mathrm{a}}$ ed. Rio de Janeiro: Elsevier; 2005. p. 297-332.

9. Villas Bôas PJF, Ruiz T. Ocorrência de infecção hospitalar em idosos internados em hospital universitário. Rev Saúde Pública. 2004;38(3):372-8.

10. Toneto MG, Moreira LF, Jeckel Neto E, Souza HP. Gastrectomia em pacientes idosos: análise dos fatores relacionados a complicações e mortalidade. Rev Col Bras Cir. 2004;31(6):373-9

11. Joia Neto LJ, Thomsom JC, Cardoso JR. Complicações repiratórias no pós-operatório de cirurgias eletivas e de urgência e emergência em um hospital universitário. J Bras Pneumol. 2005;31(1):41-7.

12. Fernandes CR, Ruiz Neto PP. O sistema respiratório e o idoso: implicações anestésicas. Rev Bras Anestesiol. 2002;52(4):461-70.

13. Netto MP, Brito FC. Urgências em geriatria. São Paulo: Atheneu; 2001.

14. Werner H, Kuntsche J. Infection in the elderly: what is different? Gerontol Geriatr. 2000; 33(5):350-8.

15. Hammerschmidt KSA. O cuidado gerontológico de enfermagem subsidiando o empoderamento do idoso com diabetes mellitus [dissertação]. Curitiba (PR): Universidade Federal do Paraná; 2007.

16. Leite MT, Gonçalves LHT. A enfermagem construindo significados a partir de sua interação social com idosos hospitalizados. Texto \& Contexto Enferm. 2009;18(1):10815.

17. Brasil. Lei n. 10.741, de 01 outubro de 2003. Dispõe sobre o Estatuto do Idoso e dá outras providências. Diário Oficial da União, Brasília, 03 out 2003.
18. Cardoso MAGM. O cuidar em gerontologia: uma análise etnográfica da prática dos enfermeiros [dissertação]. Porto (Portugal): Universidade do Porto; 2000 .

19. Lenardt MH, Hammerschmidt KSA, Pívaro ABR, Borghi ACS. Os idosos e os constrangimentos nos eventos da internação cirúrgica. Texto \& Contexto Enferm. 2007;16(4):737-45.

20. Lenardt MH, Borghi ACS, Seima MD, Hammerschmidt KSA, Michel T. O cuidado gerontogeriátrico em unidade de tratamento hemodialítico. Cogitare Enferm. 2009;14(1):37-43.

21. Brasil. Lei n. 8.842, de 04 janeiro de 2004. Dispõe sobre a política nacional do idoso, cria o Conselho Nacional do Idoso e dá outras providências. Diário Oficial da União, Brasília, 05 jan 1994.

22. Marin MJS, Marques APMF, Feres BOM, Saraiva AKH, Druzian, S. A atenção à saúde do idoso: ações e perspectivas dos profissionais. Rev Bras Geriatr Gerontol. 2008;11(2):245-58.

23. Santos SSC. O ensino da enfermagem gerontogeriátrica e a complexidade. Rev Esc Enferm USP. 2006;40(2):22835 .

24. Camacho ACLF. A gerontologia e a interdisciplinaridade: aspectos relevantes para a enfermagem. Rev LatinoAm Enfermagem. 2002;10(2):229-33. 